\title{
Phlebotomus (Larroussius) langeroni Nitzulescu, 1930 (Diptera, Psychodidae), espèce nouvelle pour l'Espagne
}

\author{
MARTINEZ ORTEGA E.*, CONESA GALLEGO E.* \& ROMERA LOZANO H.*
}

Summary: A NeW SANDFly FROM SPAIN: PHLEBOTOMUS (LARROUSSIUS) LANGERONI NitZUlescu, 1930 (Diptera, Psychodidae)

Phlebotomus langeroni has been found in iberian peninsula for the first time. A description of this species is done to discriminate it from the rest of the Larroussius subgenus' species, as well as a description of the localities where $P$. langeroni was caught and other associate species are enumerated in this paper.

KEY WORDS : Spain, Larroussius, Phlebotomus langeroni, phlebotomine sandflies, morphology, distribution.

A l'occasion d'une enquête entomologique, réalisée de mai à novembre 1991 et de juin à juillet 1993, dans la commune de Madrid, 14 exemplaires de Pblebotomus langeroni ont été capturés. Cette espèce n'était connue jusqu'à présent que de la Tunisie (Nitzulescu, 1930),

\section{Résumé :}

Les auteurs signalent la première mention pour l'Espagne de Phlebotomus langeroni Nitzulescu, 1930. Les zones de capture sont situées dans l'étage méditerranéen typique. Cette espèce est distinguée des autres représentantes du sous-genre Larroussius. In fine une liste réactualisée des espèces de la péninsule ibérique est proposée.

MOTS CLÉS : Espagne, Larroussius, Phlebotomus langeroni, phlébotomes, morphologie, distribution.

de la Libye (Nitzulescu, 1933), de l'Algérie (Parrot, 1937), du Maroc (Ristocelli, 1945) et de l'Egypte (El Sawaf, 1984). Elle est donc signalée pour la première fois en Espagne (fig. 1).

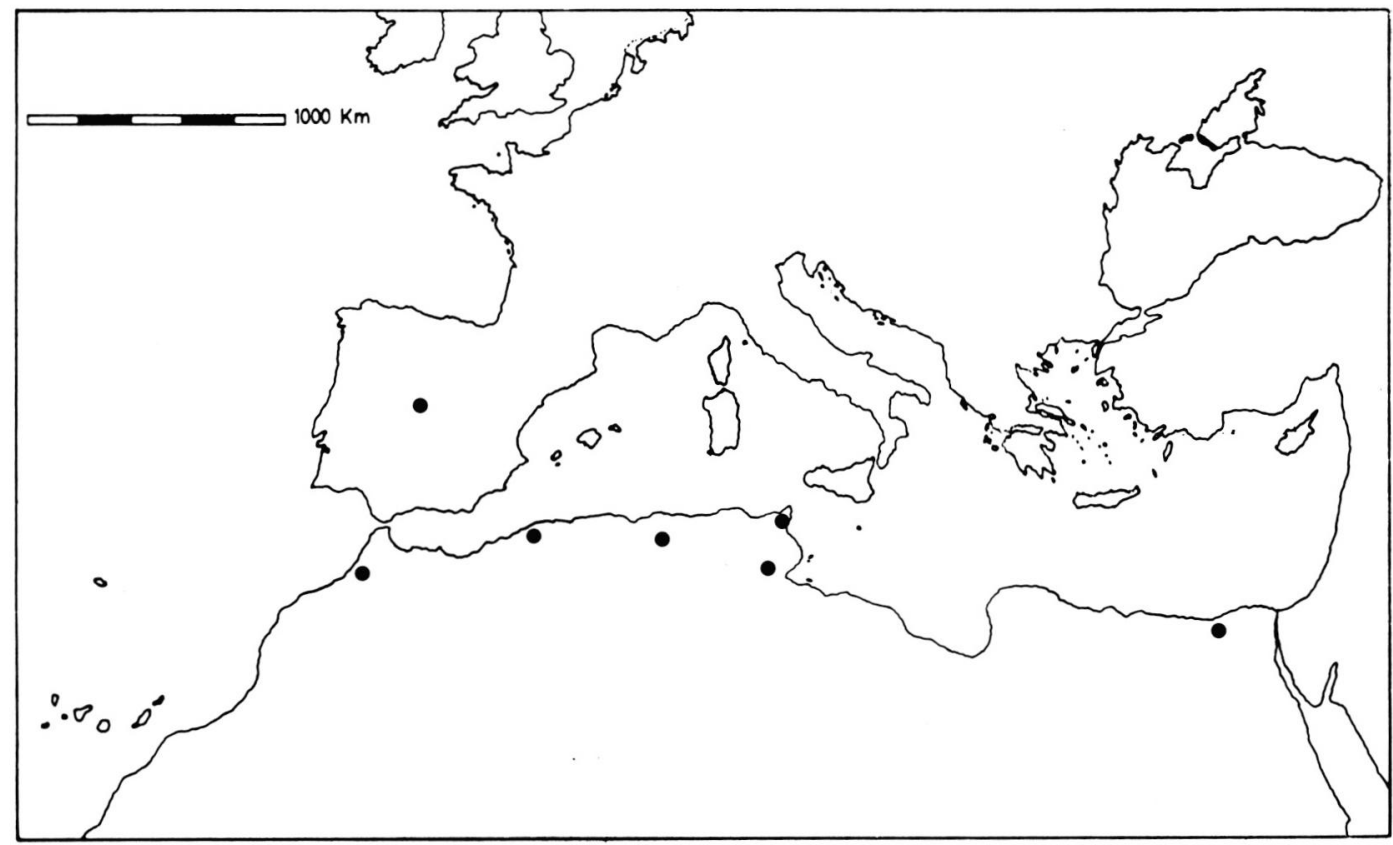

Fig. 1. - Distribution géographique de Phlebotomus langeroni.

* Departamento de Biologia Animal (Zoologia). Facultad de Biologia. Universidad de Murcia, 30071 Murcia, Espagne.
Correspondence: Ezequiel Martinez Ortega. Tel.: 34-68-307100 ext. 2370. Fax: 34-68-363963. 


\section{MÉTHODOLOGIE}

L

'échantillonnage a été effectué tous les quinze jours dans 81 stations distribuées sur l'ensemble de la commune de Madrid (climat méditerranéen sec à hiver frais). Les captures ont été réalisées par la technique des pièges adhésifs, imprégnés d'huile de ricin (Croset, 1969; Rioux et al., 1967, 1969), technique dont l'efficacité a été amplement démontrée.

Pendant les mois de juin et juillet, un complément de capture a été effectué de $21 \mathrm{~h}$ à $9 \mathrm{~h}$, à l'aide de pièges lumineux de type CDC.

\section{RÉSULTATS}

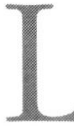

'enquête ainsi réalisée a permis de capturer douze exemplaires mâles et deux exemplaires femelles de Pblebotomus langeroni, et ce, dans les cinq localités suivantes (tableau I) :

- Camarma de Esteruelas : Tunnel construit en briques. Dépôts de carbonates sur les parois. A l'extrémité de la galerie, présence d'un puits communiquant avec l'extérieur.

- Villarejo de Salvanés I : Ferme (porcs, vaches, chiens); cultures de céréales.

- Rivas Vaciamadrid : Grottes marno-calcaires. A l'extérieur, traces de Lapins et de Rapaces.

- Villa del Prado : Vaste exploitation agricole avec écurie (moutons) et un chenil. Champ de céréales. Cours d'eau arrosant les vergers des alentours.

- Boadilla del Monte : Ferme étendue; écuries (ovins), poulailliers, chenil. Propriété située à $400 \mathrm{~m}$ du bourg de Boadilla del Monte. Dans cette localité, P. langeroni a été capturé à l'aide du piège CDC.

\section{DISCUSSION}

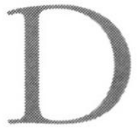

u point de vue morphologique, $P$. langeroni mâle présente des genitalia caractéristiques du sous-genre Larroussius (fig. 2.1) : style à cinq épines dont deux apicales. La différence avec les autres espèces du sous-genre réside dans la structure des valves péniennes terminées ici en pointe unique courte et assymétrique (fig. 2.2). Chez $P$. perniciosus, ces valves sont bifurquées à l'apex. Chez $P$. longicuspis, elles sont terminées en pointe unique, longue et courbée. Chez $P$. ariasi, elles présentent un aspect en battant de cloche.

$P$. langeroni femelle se distingue essentiellement par la structure de la spermathèque (fig. 2.3). Le réservoir, constitué de 9 à 10 anneaux, se poursuit par un conduit terminé en une dilatation fusiforme caractéristique (El Sawaf, 1985). Cette structure est bien dif-

\begin{tabular}{|c|c|c|c|c|}
\hline Localités & Dates & Espèces & Mâles & Femelles \\
\hline \multirow[t]{4}{*}{$\begin{array}{l}\text { Camarma } \\
\text { de } \\
\text { Esteruelas }\end{array}$} & $26 / 7 / 91$ & $\begin{array}{l}\text { P. ariasi } \\
\text { P. perniciosus } \\
\text { P. langeroni }\end{array}$ & $\begin{array}{r}1 \\
21 \\
1\end{array}$ & $\begin{array}{c}- \\
1 \\
-\end{array}$ \\
\hline & $12 / 8 / 91$ & $\begin{array}{l}\text { P. ariasi } \\
\text { P. perniciosus } \\
\text { P. langeroni }\end{array}$ & $\begin{array}{r}5 \\
55 \\
3\end{array}$ & $\begin{array}{c}- \\
1 \\
-\end{array}$ \\
\hline & 28/8/91 & $\begin{array}{l}P . \text { ariasi } \\
P . \text { perniciosus } \\
P . \text { longicuspis } \\
P . \text { langeroni }\end{array}$ & $\begin{array}{r}8 \\
58 \\
2 \\
1\end{array}$ & $\begin{array}{l}- \\
5 \\
- \\
-\end{array}$ \\
\hline & $30 / 9 / 91$ & $\begin{array}{l}\text { P. ariasi } \\
\text { P. perniciosus } \\
\text { P. langeroni }\end{array}$ & $\begin{array}{r}6 \\
19 \\
-\end{array}$ & $\begin{array}{c}1 \\
- \\
1\end{array}$ \\
\hline $\begin{array}{l}\text { Villarejo } \\
\text { de } \\
\text { Salvanés I }\end{array}$ & $15 / 8 / 91$ & $\begin{array}{l}\text { P. papatasi } \\
\text { P. perniciosus } \\
\text { P. langeroni }\end{array}$ & $\begin{array}{r}1 \\
11 \\
1\end{array}$ & $\begin{array}{c}- \\
5 \\
-\end{array}$ \\
\hline $\begin{array}{l}\text { Boadilla } \\
\text { del } \\
\text { Monte II }\end{array}$ & $30 / 6 / 93$ & $\begin{array}{l}\text { S. minuta } \\
\text { P. perniciosus } \\
\text { P. langeroni }\end{array}$ & $\begin{array}{c}- \\
6 \\
1\end{array}$ & $\begin{array}{c}1 \\
4 \\
-\end{array}$ \\
\hline $\begin{array}{c}\text { Rivas } \\
\text { de } \\
\text { Vaciamadrid }\end{array}$ & $21 / 6 / 91$ & $\begin{array}{l}\text { S. minuta } \\
P . \text { ariasi } \\
P . \text { perniciosus } \\
P . \text { langeroni }\end{array}$ & $\begin{array}{r}1 \\
4 \\
33 \\
1\end{array}$ & $\begin{array}{l}- \\
- \\
- \\
-\end{array}$ \\
\hline \multirow[t]{3}{*}{$\begin{array}{c}\text { Villa } \\
\text { del Prado }\end{array}$} & $9 / 7 / 91$ & $\begin{array}{l}\text { S. minuta } \\
\text { P. papatasi } \\
\text { P. sergenti } \\
\text { P. longicuspis } \\
\text { P. perniciosus } \\
\text { P. langeroni }\end{array}$ & $\begin{array}{r}27 \\
- \\
2 \\
2 \\
329 \\
1\end{array}$ & $\begin{array}{c}42 \\
1 \\
- \\
- \\
16 \\
-\end{array}$ \\
\hline & $25 / 7 / 91$ & $\begin{array}{l}S . \text { minuta } \\
P . \text { perniciosus } \\
P . \text { langeroni }\end{array}$ & $\begin{array}{r}6 \\
31 \\
2\end{array}$ & $\begin{array}{r}37 \\
3 \\
-\end{array}$ \\
\hline & $26 / 8 / 91$ & $\begin{array}{l}\text { S. minuta } \\
P . \text { perniciosus } \\
P . \text { langeroni }\end{array}$ & $\begin{array}{r}20 \\
51 \\
1\end{array}$ & $\begin{array}{r}31 \\
7 \\
1\end{array}$ \\
\hline
\end{tabular}

Tableau I.

férente chez les autres Larroussius présents en Espagne. Chez P. longicuspis (fig. 3.2), le conduit est pourvu à son extrémité distale d'une "formation glandulaire " réfringente, à lumière large et cordiforme, contenant de fines cloisons; chez $P$. perniciosus (fig. 3.1), celle-ci est ovalaire, à lumière étroite; chez $P$. ariasi elle est absente. Seul le tiers inférieur du conduit présente une structure morphognomonique : il se dilate en une vaste ampoule à paroi finement réticulée et débouche sur un orifice commun.

Dans les localités les plus riches en $P$. langeroni, l'espèce est exclusivement accompagnée des représentants du sous-genre Larroussius, vecteurs habituels de Leishmania infantum en Méditerranée occidentale. 


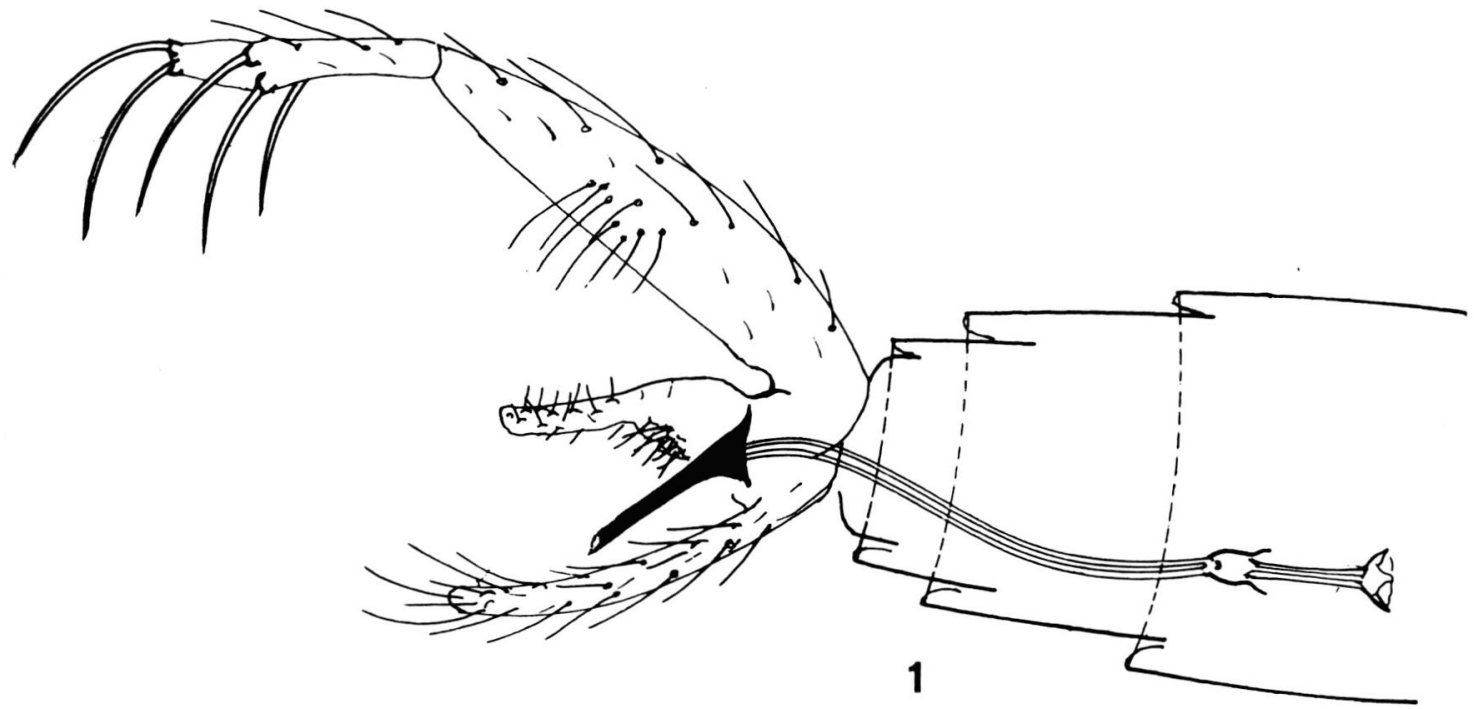

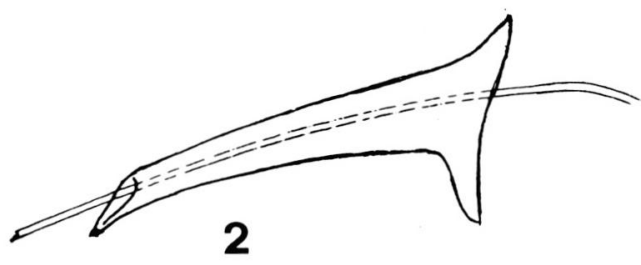

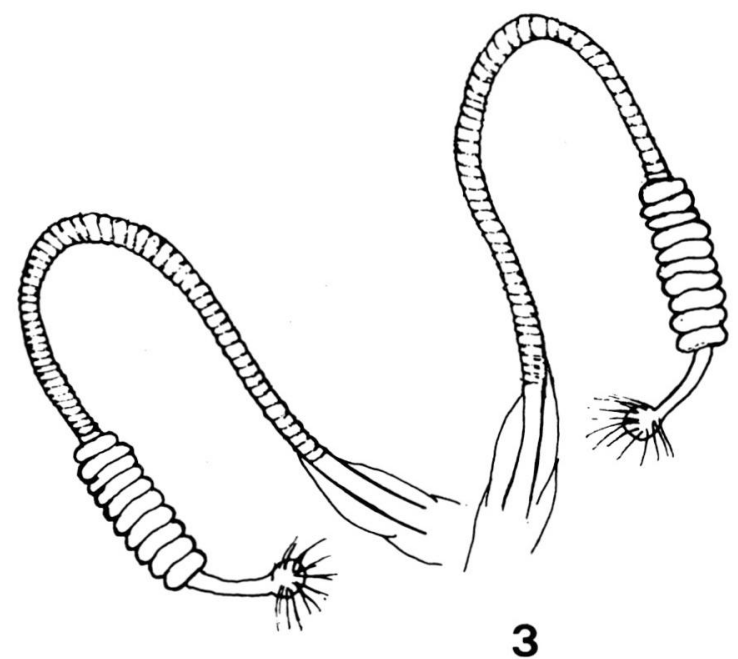

3

Fig. 2. - Genitalia de Pblebotomus langeroni
Dans les autres, s'ajoutent $P$. papatasi et Sergentomyia minuta. En Egypte la cohabitation avec $P$. papatasi est habituelle (El Said et al., 1986). Dans ce pays P. langeroni est considéré comme le vecteur putatif de L. infantum (El Sawaf et al., 1989).

Dans les localités espagnoles, les imagos de $P$. langeroni apparaissent en juin et disparaissent en septembre.

Avec ce nouveau taxon la liste des phlébotomes de la péninsule ibérique totalise les dix espèces suivantes : Sergentomyia (Sergentomyia) minuta (Rondani, 1843), Phlebotomus (Phlebotomus) papatasi (Scopoli, 1786), Phlebotomus (Adlerius) mascitii (Grassi, 1908),

Phlebotomus (Paraphlebotomus) alexandri (Sinton, 1928),

Phlebotomus (Paraphlebotomus) chabaudi (Croset et al., 1970),

Phlebotomus (Paraphlebotomus) sergenti (Parrot, 1917), Phlebotomus (Larroussius) ariasi (Tonnoir, 1921),

Phlebotomus (Larroussius) langeroni (Nitzulescu, 1930), Phlebotomus (Larroussius) longicuspis (Nitzulescu, 1930),

Phlebotomus (Larroussius) perniciosus (Newstead, 1911).

\section{REMERCIEMENTS}

Nous remercions Monsieur Rioux pour avoir rédigé le texte en français. 


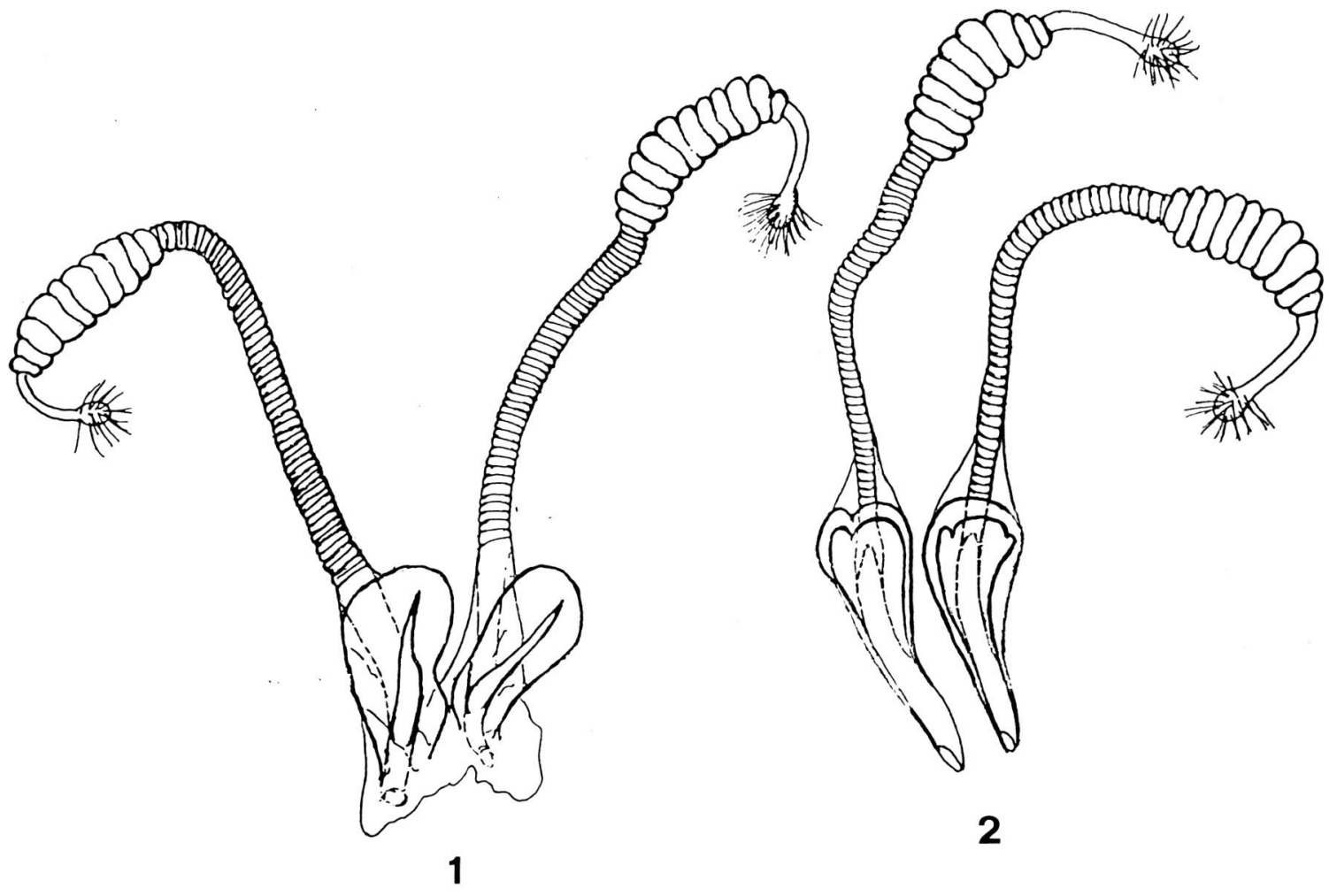

Fig. 3. - Spermathèques de $P$. perniciosus (1) et $P$. longicuspis (2).

\section{RÉFÉRENCES}

Croset H. Écologie et systématique des Pblebotomini (Diptera, Psychodidae) dans deux foyers, français et tunisien, de leishmaniose viscérale. Essai d'interprétation épidémiologique. Thèse Sciences, Montpellier, 1969, 516 p.

El Said S.M., Beier J.C., El Sawaf B.M., Doha S. \& El Kordy E. Sandflies (Diptera, Psychodidae) associated with visceral leishmaniasis in El Agamy, Alexandria Governorate, Egypt. II. Field behavior. Joumal of Medical Entomology, 1986, 23 (6), 609-615.

El Sawaf B.M., Kassem H.A. \& El SaId S.M. Description of the hitherto unknown female of Phlebotomus langeroni (Diptera, Psychodidae). Journal of Medical Entomology, 1985, 22 (3), 312-314.

El Sawaf B.M., Mansour N.S., El Said S.M., Daba S., Youssef F.G., Kenawy M.A. \& BeIER J.C. Feeding patterns of Phlebotomus papatasi and Pblebotomus langeroni (Diptera, Psychodidae), in El Agamy, Egypt. Journal of Medical Entomology, 1989, 26 (5), 497-498.

El Sawaf B.M., Beier J.C., Hussein S.M., Kassem H.A. \& Satier S.A. Phlebotomus langeroni: a potential vector of Kala-azar in the Arab Republic of Egypt. Transactions of the Royal Society of Tropical Medicine and Hygiene, 1984, 78, 421.
Nitzulescu V. Phlebotomus langeroni n. sp. Phlebotomus langeroni var. longicuspis n. var. de Douart-shott (Tunisie). Annales de Parasitologie Humaine et Comparée, 1930, 8, 547-553.

Nitzulescu V. Essai de classification des phlébotomes. Annales de Parasitologie Humaine et Comparée, 1931, 9, 271-275.

PARRot L. Notes sur les phlébotomes. XX. Sur Phlebotomus langeroni var. longicuspis Nitzulescu, 1930. Archives de l'Institut Pasteur d'Algérie, 1936, 14, 137-143.

Rioux J.A., Golvan Y.J., Croset H., Houin R., Juminer B., Bain O. \& Tour S. Écologie des leishmanioses dans le sud de la France. 1. Les phlébotomes. Échantillonnage-Éthologie. Annales de Parasitologie Humaine et Comparée, $1967,42,561-603$.

Rioux J.A. \& Golvan Y.J. Épidémiologie des leishmanioses dans le sud de la France. Monographie INSERM, 1969, 37, 223 p.

Ristocelli A. Contribution à l'étude des phlébotomes du Maroc. Annales de Parasitologie Humaine et Comparée, 1939, 17, 364-365.

Reçu le 2 février 1995 Accepté le 13 novembre 1995 